\title{
A Novel Vaccine for The Novel Corona Virus
}

\author{
Robert 0 Dillman*, Gabriel I Nistor, Aleksandra J Poole, Candace Hsieh and Hans S Keirstead \\ AIVITA Biomedical, Inc., USA \\ *Corresponding author: Robert 0 Dillman, AIVITA Biomedical, Inc. Irvine, California, USA. \\ To Cite This Article: Robert O Dillman, Gabriel I Nistor, Aleksandra J Poole, A Novel Vaccine for The Novel Corona Virus. Am J Biomed Sci \& Res. \\ 2020 - 11(3). AJBSR.MS.ID.001631. DOI: 10.34297/AJBSR.2020.11.001631.
}

Received: 阱 November 11, 2020; Published: 眥 December 18, 2020

\section{Opinion}

A high level of "herd immunity" will be necessary to control the current COVID-19 pandemic caused by the SARS-CoV-2 virus. Herd immunity is achieved when a substantial majority of a population becomes immune to a disease, making the spread of the pathogen unlikely. Herd immunity will eventually be achieved by the combination of individuals who recover from active infection, and preventive immunization of unaffected individuals. Because of the extensive morbidity and mortality associated with active infection, preventive vaccination is the preferred strategy.

Multiple vaccines have been developed and are being tested in clinical trials, including (1) inactivated (dead) SARS-CoV-2 virus (SinoVac, Sinopharm), (2) attenuated (weakened) SARS-CoV-2 virus, (3) viral DNA (Sanofi-Glaxo-Smith-Kline), (4) viral RNA (Moderna, Biontech-Pfizer) (5) SARS-CoV-2 genes expressed in a replicating virus (Johnson \& Johnson, Sanofi) (6) SARS-CoV-2 genes expressed in a non-replicating virus (CanSino, AstraZeneca-Oxford), (7) SARSCoV-2 proteins (Nanovax, Novavax), or (8) a virus-like particle that contains no viral material, but may look like the virus to the immune system. After injection, all of these approaches rely on ingestion and digestion of the antigen by endogenous antigen presenting cells (APC), which break down the protein into 9 to 25 amino acid peptides for presentation to lymphocytes. APC communicate with lymphocytes via their antigen-specific receptors for specific clonal activation that results in B-cell mediated humoral immunity (antibodies and Th2 response), T-cell immunity (cytotoxic T cells and Th1 response), and memory for an accelerated response in the event of future exposure or re-exposure to the actual pathogen. Many of these vaccines include an immune-enhancing adjuvant to increase the attraction of APCs to the injection site. Each of these vaccines is mass-produced so that the same product can be injected into each individual.
What might be considered a "novel" approach to the "novel" corona virus is a subject-specific personalized SARS-CoV-2 dendritic cell vaccine. Dendritic cells (DC) are considered to be the most important of the APC for initiating the host immune response to specific antigens. The advantage of this approach is that each subject's DC are incubated with the viral antigen ex vivo, thus bypassing the first critical in vivo step for activating the immune system. The SARS-CoV-2 antigens, especially the spike protein that contains the receptor-binding domain for the angiotensin converting enzyme (ACE) receptor have been sequenced and cloned to produce large quantities of recombinant protein. Procedures for manufacturing DC from peripheral blood mononuclear cells (PBMC) and cryopreservation of such products are well-established. DCs loaded with cancer antigens have been tested as a form of anticancer immunotherapy for more than two decades. Hundreds of thousands to a few million DC can be generated from the PBMC contained in a 40 to $50 \mathrm{ml}$ blood sample. Recombinant antigen is incubated with each subject's DC ex vivo to produce the vaccine. The theoretical advantages of this approach include: (1) the efficiency of ex vivo loading of antigen into the DC, (2) the small quantity of antigen required, and (3) jump-starting the immune cascade by injecting large numbers of the subject's own antigen-loaded DC. The superiority of injecting DCs that have been loaded with antigen ex vivo, compared to direct injections of antigen, has been proven in the treatment of cancer patients based on immune response, and survival benefit. A SARS-CoV-2 DC vaccine may be more effective and better tolerated than any other COVID-19 vaccines.

This approach could be applied to create personalized preventive vaccines against any pathogen. In animal models, DCbased vaccines have demonstrated protection against leishmaniasis, Herpes simplex virus, influenza virus, Candida albicans and HIV. 
Application of DC immunotherapy as prophylaxis for infectious diseases is both scientifically rational and potentially cost-effective, especially for populations who are at risk of increased morbidity and respond poorly to traditional vaccination.

There are obvious challenges to such a personalized approach. First is the scale of manufacturing required. Instead of manufacturing enormous quantities of a uniform vaccine product for mass distribution, this approach requires manufacturing a new product for each specific individual. This would require "scaling up" capacity within a manufacturing site as well as "scaling out" capacity by adding additional manufacturing sites. This also could be accomplished by providing regional laboratories with individual small manufacturing devices containing, media, cytokines, and antigen, to which the subject's PBMC would be added. DC differentiation and antigen processing would occur during a few days of incubation. Each product could be provided in a vial and/ or drawn into a syringe for injection. The second major challenge to this approach is regulatory. Good manufacturing practices (GMP) and sterile environments are a prerequisite for manufacturing biological products. However, the testing of the final subject-specific product requires a different paradigm. When one is manufacturing large batches of chemicals, cytokines, or monoclonal antibodies, for thousands to millions of doses, it is critical that such products do not contain potentially harmful agents. For this reason, extensive testing is performed to exclude possible contaminants including tests for viruses, mycoplasma, endotoxin, and United States Pharmacopeial (USP) convention for sterility prior to release of such products. Such testing is expensive, but necessary because of the potential risk to thousands or millions of individuals who might receive a contaminated product generated in a single batch, and the cost per dose gets lower and lower as the number of doses in a batch is increased. However, from a risk-to-benefit perspective, such testing is of little value for subject-specific products, economically impractical from a cost-to-benefit perspective and reduces the quantity of the treatment product because of the need to remove a portion of the final product for safety testing. For this subject-specific product, there is risk to only one subject. The cells introduced are autologous, and the other intermediate components of the product: media, cytokines, antigen, must meet USP standards for batch production, which takes place in a sterile environment. As long as each subject receives his/her own product, there is potential benefit and minimal risk.

\section{Acknowledgement}

None.

\section{Conflict of Interest}

No conflict of interest. 